\title{
Consequences of Using an Orthotropic Stress Tensor for Left Ventricular Systole
}

\author{
Tobias Gerach, Steffen Schuler, Ekaterina Kovacheva, Olaf Dössel, Axel Loewe \\ Institute of Biomedical Engineering, Karlsruhe Institute of Technology (KIT), Karlsruhe, Germany
}

\begin{abstract}
A variety of biophysical and phenomenological active tension models has been proposed during the last decade that show physiological behaviour on a cellular level. However, applying these models in a whole heart finite element simulation framework yields either unphysiological values of stress and strain or an insufficient deformation pattern compared to magnetic resonance imaging data.

In this study, we evaluate how introducing an orthotropic active stress tensor affects the deformation pattern by conducting a sensitivity analysis regarding the active tension at resting length $T_{\mathrm{ref}}$ and three orthotropic activation parameters $\left(K_{\mathrm{ss}}, K_{\mathrm{sn}}\right.$ and $\left.K_{\mathrm{nn}}\right)$. Deformation of left ventricular contraction is evaluated on a truncated ellipsoid using four features: wall thickening (WT), longitudinal shortening $(L S)$, torsion $(\Theta)$ and ejection fraction $(E F)$.

We show that EF, WT and LS are positively correlated with the parameters $T_{\mathrm{ref}}$ and $K_{\mathrm{nn}}$ while $K_{\mathrm{ss}}$ reduces all of the four observed features. Introducing shear stress to the model has little to no effect on EF, WT and LS, although it reduces torsion by up to $3^{\circ}$.

We find that added stress in the normal direction can support healthy deformation patterns. However, the twisting motion, which has been shown to be important for cardiac function, reduces by up to $20^{\circ}$.
\end{abstract}

\section{Introduction}

Modern magnetic resonance imaging (MRI) methods have become a widespread clinical tool to diagnose function as well as malfunction of cardiac mechanics especially in the left ventricle (LV). It makes the non-invasive assessment of in-vivo ventricular wall motion possible and opens a window for an interpretation of the data with regards to the development of local wall stress. Additionally, MRI simultaneously serves as the main data foundation and validation tool for in-silico studies.

To simulate the aforementioned local wall stresses with finite element (FE) techniques, there exists a wide vari- ety of cellular tension development models based on measured human or animal tissue preparations that can be divided into two main groups: biophysical models based on physiological first principles or phenomenological models. The latter have shown to be able to reproduce the deformation patterns observed in MRI data. However, most of the time this is only possible whilst neglecting physiological properties such as the dependence of active stress on fiber stretch or the range of fiber stretch the model produces during ventricular systole. Biophysical models on the other hand, do not produce the amount of stress necessary to accomplish deformation patterns similar to those observed in MRI data due to the lower force generation during contraction.

As a potential approach to overcome these problems, we implement an active stress tensor including components in the sheet and normal direction with added shear stress similar to $[1,2]$ in our existing FE framework . This is based on experimental data that show a simultaneous development of fiber, cross-fiber and shear stresses in a barium contracted rabbit myocardium by Lin \& Yin [3]. They showed that the stress in cross-fiber or normal direction $\mathbf{S}\left(\mathbf{n}_{0}, \mathbf{n}_{0}\right)$ lies between 20 and $62 \%$ of the stress in fiber direction $\mathbf{S}\left(\mathbf{f}_{0}, \mathbf{f}_{0}\right)$. To assess how this new form of an active stress tensor changes the deformation patterns of the LV, we run a sensitivity study including four parameters and observe how they change major biomarkers during contraction: wall thickness, longitudinal shortening, torsion and ejection fraction. Finally, we compare our results to clinically reported values of healthy subjects.

\section{Materials and methods}

\subsection{Idealized left ventricular geometry}

A truncated ellipsoid obtained from a cardiac mechanics benchmark [4] is used to represent an idealized left ventricle. The mesh is scaled by a factor of 3.75 to obtain a more realistic end-diastolic volume (EDV) of $133.55 \mathrm{ml}$ (fig. 1). Furthermore, the ellipsoid is closed at the base to represent the mitral valve. For the discretization, a total number of $\approx 8 \mathrm{k}$ quadratic tetrahedra with $\approx 13.5 \mathrm{k}$ nodes is 
used. Fiber $\mathbf{f}_{0}$, sheet $\mathbf{s}_{0}$, and (sheet-)normal $\mathbf{n}_{0}$ directions were calculated on a high-resolution LV geometry using the rule based algorithm presented in [5] $\left(\alpha_{e p i}=-70^{\circ}\right.$, $\alpha_{\text {endo }}=80^{\circ}, \beta_{\text {epi }}=\beta_{\text {endo }}=0^{\circ}$ ) and interpolated onto the 5 Gaussian quadrature points of each element of the tetrahedral mesh used in this study.

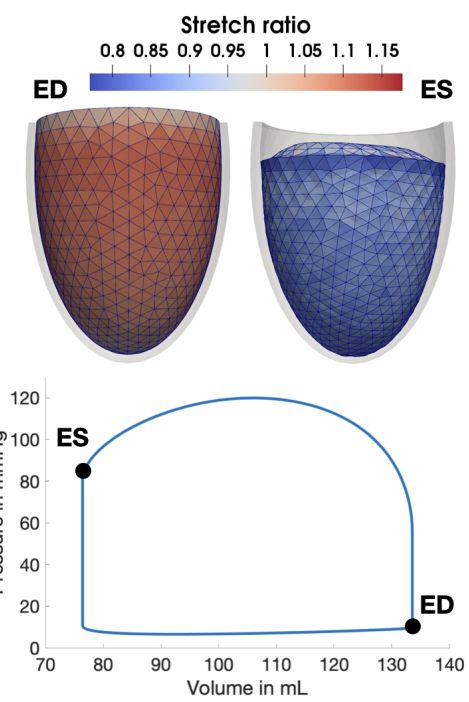

Figure 1. Bottom: pV-loop of the LV with ED and ES marked. Top: corresponding ED and ES state of the FE mesh with the colorbar showing the fiber stretch ratio $\lambda_{f}$.

\subsection{Modelling ventricular biomechanics}

Modelling of cardiac mechanics is done using the CardioMechanics software [6]. The kinematics are determined by solving the governing equation for the balance of linear momentum in total Lagrangian formulation. The tissue is assumed to be hyperelastic, transversely isotropic and nearly incompressible as described by the constitutive model by Guccione et al. [7] with $C=264 \mathrm{~Pa}, b_{f}=8.5$, $b_{t}=3.4$ and $b_{f s}=5.95$. Quasi incompressibility is enforced by adding a penalty term:

$$
W_{\mathrm{Vol}}=\frac{K}{2}(\operatorname{det} \mathbf{F}-1)^{2},
$$

with $K=1 \mathrm{MPa}$ to the constitutive equation. This value keeps the volumetric changes of the tissue below $10 \%$. Time integration is performed using the Newmark-beta scheme with $\beta=0.3$ and $\gamma=0.6$. The non-linear system is solved using Newton's method and LU decomposition within the PETSc framework [8]. An active stress formulation is used, allowing an additive decomposition of the second Piola-Kirchoff stress tensor into passive and active parts:

$$
\mathbf{S}=\mathbf{S}_{\mathrm{pas}}+\mathbf{S}_{\mathrm{act}}
$$

The active stress $\mathbf{S}_{\text {act }}$ is chosen to be an orthotropic stress tensor in the form of

$$
\mathbf{S}_{\mathrm{act}}=T_{\mathrm{a}} \cdot\left(\begin{array}{ccc}
1 & 0 & 0 \\
0 & K_{\mathrm{ss}} & K_{\mathrm{sn}} \\
0 & K_{\mathrm{ns}} & K_{\mathrm{nn}}
\end{array}\right),
$$

where $T_{\mathrm{a}}$ is the cellular active tension, and $K_{\mathrm{ss}}, K_{\mathrm{sn}}, K_{\mathrm{ns}}$ as well as $K_{\mathrm{nn}}$ are orthotropic activation parameters. Active tension development on a cellular level was determined using the model described by Land et al. [9]. This model incorporates a dependence on fiber stretch ratio $\lambda_{f}$ and is driven by an experimentally measured calcium transient.

As boundary conditions, a frictionless and permanent contact handling problem is solved between the epicardial surface of the LV and the inner surface of an additional ellipsoid mesh as described in [6]. On the outer surface of this surrounding ellipsoid, Dirichlet boundary conditions are applied. The pressure within the LV is determined by a closed-loop lumped parameter model of circulation that is strongly coupled to the FE model by keeping the absolute chamber volumes $V_{\mathrm{FE}}$ and $V_{\text {circ }}$ equal [10]. The resulting pressure in the LV is applied uniformly to the endocardial surface.

A pre-stressed end-diastolic state is achieved by finding the pressure free state using the method described by Bols et al. [11]. Afterwards, the LV is inflated by applying a pressure of $7.5 \mathrm{mmHg}$ to the endocardial surface to obtain the pre-stretched end-diastolic configuration.

\subsection{Mechanical biomarkers}

There are typically four distinct features that can be observed during ventricular contraction: wall thickening, longitudinal shortening, torsion and ejection fraction. Wall thickening (WT) is the relative displacement between points on the endocardium and epicardium at the same position relative to the heart's long axis

$$
\mathrm{WT}=100 \frac{\mathrm{T}-\mathrm{T}_{0}}{\mathrm{~T}_{0}},
$$

where $\mathrm{T}$ and $\mathrm{T}_{0}$ are the mean wall thicknesses during endsystole and end-diastole calculated using the Eulerian PDE approach introduced in [12]. Longitudinal shortening (LS) is calculated as the fractional change in distance between the endocardial base and apex from ED $\left(\mathrm{L}_{0}\right)$ to ES $(\mathrm{L})$

$$
\mathrm{LS}=100 \frac{\mathrm{L}_{0}-\mathrm{L}}{\mathrm{L}_{0}} .
$$

Torsion $\Theta$ is approximated as the net difference between apical and basal rotation from two short axis slices during ES using

$$
\cos (\Theta)=\frac{\mathbf{r}_{\text {Base }} \cdot \mathbf{r}_{\text {Apex }}}{\left\|\mathbf{r}_{\text {Base }}\right\| \cdot\left\|\mathbf{r}_{\text {Apex }}\right\|},
$$


where $\mathbf{r}_{\text {Base }}$ and $\mathbf{r}_{\text {Apex }}$ are points on the basal and apical epicardium respectively. Lastly, ejection fraction (EF) is evaluated in this study using the end-diastolic volume (EDV) and the end-systolic volume (ESV) from the lumped parameter model of the circulatory system:

$$
\mathrm{EF}=100 \frac{\mathrm{EDV}-\mathrm{ESV}}{\mathrm{EDV}}
$$

Physiological ranges of these four mechanical biomarkers are shown in table 1.

Table 1. Reference values observed in healthy subjects.

\begin{tabular}{llll}
\hline \hline Mechanical biomarker & Values & Unit & Ref \\
\hline WT & $18-100$ & $\%$ & {$[13]$} \\
LS & $13-21$ & $\%$ & {$[14]$} \\
$\Theta$ & $13-27$ & $\mathrm{deg}$ & {$[15]$} \\
$\mathrm{EF}$ & $48-69$ & $\%$ & {$[16]$} \\
\hline \hline
\end{tabular}

\subsection{Sensitivity analysis}

To investigate how sensitive the mechanical biomarkers are to changes in the parameters of an orthotropic stress tensor in the form of equation (3), the parameters $T_{\text {ref }}$, $K_{\mathrm{ss}}, K_{\mathrm{sn}}, K_{\mathrm{ns}}$ and $K_{\mathrm{nn}}$ are varied using a one-at-a-time (OAAT) approach. Here, $T_{\text {ref }}$ refers to the maximal active tension at resting length in the Land et al. tension model with a default value of $120 \mathrm{kPa}$. The baseline value for the other parameters is set to $K_{\mathrm{ss}}=K_{\mathrm{sn}}=K_{\mathrm{nn}}=0$ with $K_{\mathrm{sn}}=K_{\mathrm{ns}}$ at all times. For the OAAT, ten values per parameter are determined by the lower and upper bounds $A$ and $B$ of the parameter ranges summarized in table 2 through

$$
x_{i, j}=A_{i}+(j-1) \frac{B_{i}-A_{i}}{n_{\mathrm{val}}-1}, \quad i=1,2,3,4
$$

where $j=1,2,3, \ldots, n_{\text {val }}$ will be referred to as case number with $n_{\text {val }}=10$.

Table 2. Parameter ranges for the sensitivity study.

\begin{tabular}{llll}
\hline \hline Parameter & Range of values & Unit & Ref. \\
\hline$T_{\text {ref }}$ & $80-440$ & $\mathrm{kPa}$ & {$[9]$} \\
$K_{\mathrm{ss}}$ & $0.0-0.6$ & - & {$[1,3]$} \\
$K_{\mathrm{sn}}$ & $0.0-0.07$ & - & {$[1]$} \\
$K_{\mathrm{nn}}$ & $0.0-0.6$ & - & {$[1,3]$} \\
\hline \hline
\end{tabular}

\section{Results}

The biomarker sensitivity study using the OAAT approach is shown in figure 2. The $\mathrm{pV}$-loop and deformation pattern during ED and ES for a reference simulation with
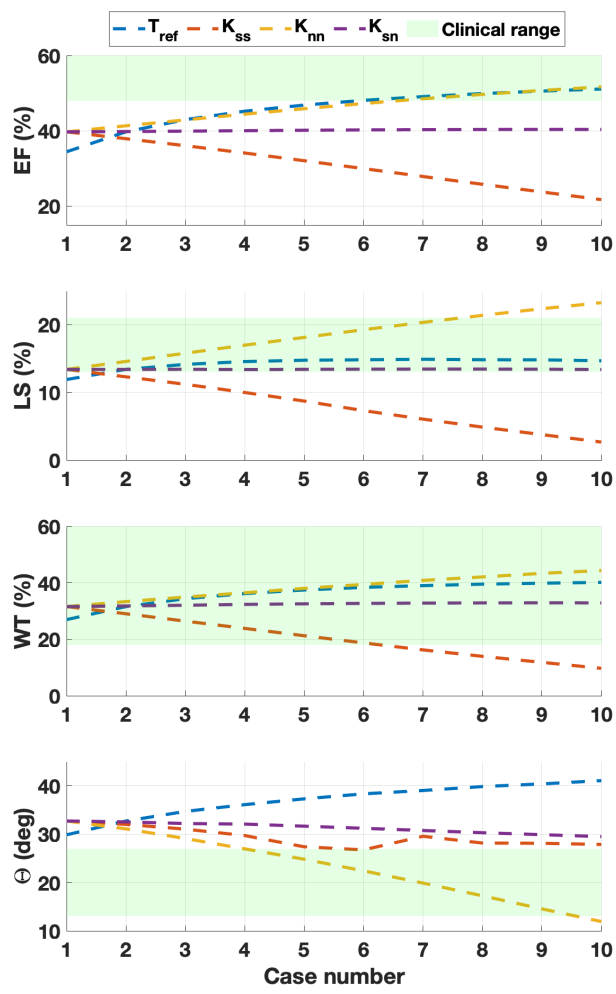

Figure 2. Change of EF, LS, WT, and $\Theta$ depending on the parameters $T_{\text {ref }}, K_{\mathrm{ss}}, K_{\mathrm{sn}}$ and $K_{\mathrm{nn}}$.

$T_{\text {ref }}=120 \mathrm{kPa}$ and $K_{\mathrm{ss}}=K_{\mathrm{sn}}=K_{\mathrm{nn}}=0$ is shown in figure 1. Almost all cases show either monotonically increasing or decreasing, non-linear behaviour. Increasing stress in the sheet direction $\left(K_{\mathrm{SS}}\right)$ reduces all the mechanical biomarker values by up to $50 \%$ of the initial values. The results show that physiological ranges of EF can only be reached with either high values of active tension $\left(T_{\text {ref }}\right)$ or additional tension in the normal direction $\left(K_{\mathrm{nn}}\right)$. However, the resulting EF is still at the low end of clinical reference values and increased active stress in the normal direction leads to values of LS outside the healthy range. Using higher active tension keeps LS constant above a certain threshold (case number $>3$ ) and starts to decrease again for values of $T_{\text {ref }}$ above $400 \mathrm{kPa}$. Although shear stress $\left(K_{\mathrm{sn}}\right)$ in the tissue does not affect EF, LS and WT at all, the torsion $\Theta$ decreases by up to $3^{\circ}$. An even stronger reduction of torsion can be observed through additional stress in the sheet and normal directions.

\section{Discussion}

In this study, we present a sensitivity analysis on the effect of using an orthotropic stress tensor on four ma- 
jor mechanical biomarkers characterizing LV systole. We found that varying the considered parameters either led to a monotonic increase or decrease of the evaluated mechanical biomarkers EF, LS, WT and $\Theta$. The change in $\Theta$ due to an increase in $K_{\mathrm{ss}}$ is an exception to this. However, we observed a $\pm 5^{\circ}$ increase in standard deviation due to measurement errors and asymmetries in contraction in cases 7 to 10 which can explain this behaviour.

The scaling parameter of the active tension model $T_{\text {ref }}$ was positively correlated with EF, WT, LS and $\Theta$. However, reaching healthy values of EF above $50 \%$ was only possible by choosing $T_{\text {ref }}$ larger than $320 \mathrm{kPa}$. These are unusually high values compared to typical values used in other modeling studies $\left(T_{\text {ref }}<180 \mathrm{kPa}\right)$. The other option which led to a healthy EF, was to add stress in the normal direction with up to $60 \%$ of $T_{\mathrm{a}}$. Additionally, this increased LS above clinically reported values and constrained the twisting motion of the LV. Adding stress in the sheet direction using the parameter $K_{\mathrm{ss}}$ reduced all four mechanical biomarkers by up to $50 \%$ of their initial values. The additional contraction in the sheet direction constrains the displacement of the LV by counteracting WT. The imposed nearly incompressible material behaviour forces the tissue to expand in the fiber and normal direction, hence the lower LS. Shear stress is found to be negligible with no effect on EF, LS and WT. However, we observed a $3^{\circ}$ reduction in torsion of the LV. This is not necessarily an undesirable outcome, since torsion in general is above the clinically observed range in our reference configuration.

In conclusion, we found that a stress tensor with a component in the normal direction can help in achieving a more physiological deformation pattern. In particular, choosing $0.25 \leq K_{\mathrm{nn}} \leq 0.45$ helped to keep the observed biomarkers in the range of clinical observations. Further investigation is required if and how a change in the fiber orientation or the boundary conditions affects this behaviour.

\section{Acknowledgments}

This project was funded by the Deutsche Forschungsgemeinschaft (DFG, German Research Foundation) Project-ID 258734477 - SFB 1173.

\section{References}

[1] Dorri F, et al. A finite element model of the human left ventricular systole. Computer methods in biomechanics and biomedical engineering 2006;

[2] Levrero-Florencio F, et al. Sensitivity analysis of a stronglycoupled human-based electromechanical cardiac model: Effect of mechanical parameters on physiologically relevant biomarkers. Computer Methods in Applied Mechanics and Engineering 2020;

[3] Lin DHS, Yin FCP. A multiaxial constitutive law for mammalian left ventricular myocardium in steady-state barium contracture or tetanus. Journal of Biomechanical Engineering 1998;

[4] Land S, et al. Verification of cardiac mechanics software: benchmark problems and solutions for testing active and passive material behaviour. Proc R Soc A 2015;

[5] Bayer JD, et al. A novel rule-based algorithm for assigning myocardial fiber orientation to computational heart models. Annals of Biomedical Engineering 2012;

[6] Fritz T, et al. Simulation of the contraction of the ventricles in a human heart model including atria and pericardium : Finite element analysis of a frictionless contact problem. Biomechanics and Modeling in Mechanobiology 2014;

[7] Guccione JM, et al. Finite element stress analysis of left ventricular mechanics in the beating dog heart. J Biomechanics 1995;

[8] Balay S, et al. PETSc users manual. Technical Report ANL95/11 - Revision 3.12, Argonne National Laboratory, 2019.

[9] Land S, et al. A model of cardiac contraction based on novel measurements of tension development in human cardiomyocytes. Journal of Molecular and Cellular Cardiology 2017; 106:68 - 83.

[10] Kerckhoffs RCP, et al. Coupling of a 3d finite element model of cardiac ventricular mechanics to lumped systems models of the systemic and pulmonic circulation. Annals of Biomedical Engineering 2007;

[11] Bols J, et al. A computational method to assess the in vivo stresses and unloaded configuration of patient-specific blood vessels. Journal of Computational and Applied Mathematics 2013;

[12] Yezzi AJ, Prince JL. An eulerian PDE approach for computing tissue thickness. IEEE transactions on medical imaging 2003;

[13] Sechtem U, et al. Regional left ventricular wall thickening by magnetic resonance imaging: Evaluation in normal persons and patients with global and regional dysfunction. The American Journal of Cardiology 1987;

[14] Emilsson K, et al. Mitral annulus motion versus long-axis fractional shortening. Experimental Clinical Cardiology 2006;.

[15] Kocabay G, et al. Normal left ventricular mechanics by two-dimensional speckle-tracking echocardiography. reference values in healthy adults. Revista espanola de cardiologia English ed 2014;

[16] Petersen SE, et al. Reference ranges for cardiac structure and function using cardiovascular magnetic resonance (CMR) in caucasians from the UK biobank population cohort. Journal of cardiovascular magnetic resonance 2017;

Address for correspondence:

Tobias Gerach

Institute of Biomedical Engineering, Karlsruhe Institute of Technology (KIT), Kaiserstr. 12, 76131, Karlsruhe, Germany.

publications@ibt.kit.edu 